\title{
EL PODER SIMBÓLICO DE LA UNESCO EN AMÉRICA LATINA SOBRE EL VÍNCULO FLACSO - UNESCO
}

DOI: http://dx.doi.org/10.1590/2236-3459/72083

\author{
Anabella Abarzúa Cutroni' \\ IInstituto de Ciencias Humanas, Sociales y Ambientales (Incihusa) - Consejo Nacional de Investigaciones \\ Científicas y Técnicas (Conicet), Mendoza, Argentina
}

\section{$\cos 80$}

\begin{abstract}
Resumen
Nuestras pesquisas indican que la Unesco ha sido históricamente una Organización de alto impacto a nivel simbólico. El patrocinio de esta Organización de la ONU a determinadas iniciativas científicas, educativas y culturales no se llevó a cabo sólo mediante financiamiento, la contratación de expertos y el otorgamiento de becas sino que implicó también el aval, el crédito simbólico, de una organización internacional. Observar este fenómeno es precisamente el objetivo del presente artículo. Para esto tomamos el caso de un patrocinio emblemático que la Unesco desarrolló en América Latina desde fines de la década de 1950: la creación y sostenimiento de la Facultad Latinoamericana de Ciencias Sociales (Flacso).

Palabras claves: poder simbólico, expertos, becas.
\end{abstract}

\section{O PODER SIMBÓLICO DA UNESCO NA AMÉRICA LATINA SOBRE A LIGAÇÃO FLACSO - UNESCO}

\begin{abstract}
Resumo
Nossas pesquisas sugerem que a Unesco historicamente teve um grande impacto a nível simbólico. $\mathrm{O}$ patrocínio das iniciativas científicas, educacionais e culturais específicas da Organização das Nações Unidas realizada não só através de financiamento, a contratação de especialistas e bolsas de estudo, mas também envolveu o crédito simbólico de uma organização internacional. O objetivo deste artigo é observar este fenômeno. Para isso, nos tomamos o caso de um patrocínio porta-estandarte da Unesco desenvolvido na América Latina desde o final dos anos 1950: a criação e o apoio da Faculdade Latino-Americana de Ciências Sociais (Flacso).

Palavras-chave: poder simbólico, especialistas, bolsas de estudo.
\end{abstract}

\section{UNESCO SYMBOLIC POWER IN LATIN AMERICA ABOUT THE LINK FLACSO-UNESCO}

\begin{abstract}
Our research indicates that, historically, Unesco had been an Organization of high impact in a symbolic level. The support from this Organization of the UN to sciences, educations and cultural initiatives implies funds, experts, fellowships and, the most important, the symbolic credit of an international organization. The objective of this paper is to analyze that. Then we study an emblematic support of Unesco in Latin America: la creation and sustenance of the Latin American Faculty of Social Science (Flacso) since 1950 decade.

Keywords: symbolic power, experts, fellowships.
\end{abstract}




\section{LE POUVOIR SYMBOLIQUE DE L'UNESCO EN AMERIQUE LATINE SUR LE LIEN FLACSO - UNESCO}

\section{Résumé}

Notre recherche suggère que l'Unesco a toujours eu une forte incidence sur le plan symbolique. Le parrainage des initiatives scientifiques, éducatives et culturelles spécifiques Organisation des Nations Unies réalisée non seulement par le financement, le recrutement d'experts et des bourses, mais aussi impliqué le crédit symbolique d'une organisation internationale. L'objectif de cet article est d'observer ce phénomène. Pour cela, nous prenons le cas d'un parrainage de repère, l'Unesco a développé en Amérique latine depuis la fin des années 1950: la création et le soutien de la Faculté latino-américaine de sciences sociales (Flacso).

Mots-clés: puissance symbolique, des experts, des bourses. 


\section{Introducción}

os funcionarios internacionales de la Unesco (Organización de las Naciones Unidas para la Educación, la Ciencia y la Cultura) suelen ponderar el impacto de la Organización en materia educativa, científica y cultural en términos de éxito o fracaso según si los objetivos, planteados cuando se planificó determinada iniciativa, tuvieron una correlación lineal y precisa con los resultados obtenidos ${ }^{1}$. Sin embargo, desde un punto de vista analítico no debemos soslayar el hecho de que no todas las variables que están en juego en la implementación del programa de la Unesco están, ni estuvieron históricamente, exclusivamente en manos de los funcionarios que, desde París, comandan esta Organización. Desde una perspectiva académica intentamos morigerar esta tendencia y complejizar el análisis a partir de un estudio detallado de iniciativas puntuales que la Unesco desarrolló a escala nacional².

Nuestras pesquisas indican que la Unesco ha sido históricamente una Organización de alto impacto a nivel simbólico. El patrocinio a determinadas iniciativas científicas, educativas y culturales no se llevó a cabo sólo mediante financiamiento, la contratación de expertos y el otorgamiento de becas si no que implicó también el aval, el crédito simbólico, de una organización internacional prestigiosa. Observar este fenómeno es precisamente el objetivo del presente artículo. Para esto tomamos el caso de un patrocinio emblemático que la Unesco desarrolló en América Latina desde fines de la década de 1950: la creación y sostenimiento de la Facultad Latinoamericana de Ciencias Sociales (Flacso) en Santiago de Chile.

Poner a la Unesco en foco implica la necesidad de comprender cómo se articulaban determinados ámbitos. Lo nacional-internacional y lo político-académico fueron ejes que atravesaron y articularon permanentemente el quehacer de los funcionarios (nacionales e internacionales) y académicos que intervinieron en cada una de las iniciativas que la Unesco patrocinó desde su creación en 1945 hasta la actualidad ${ }^{3}$. El análisis de los intercambios epistolares entre estos agentes demuestra que gran parte de su tarea consistía en construir una serie de consensos tanto diplomático-políticos como académicos. Subrayar esta necesidad de consenso académico implica destacar que en la Unesco estaban en disputa el reconocimiento de la universalidad de saberes y practicas científicas, educativas y culturales particulares. Debido a las materias de competencia de esta Organización, la generación y conservación de prestigio académico y sobre todo la

\footnotetext{
1 Distintos documentos administrativos (memorias, balances de gestión, informes de misión, etc.) denotan esto que afirmamos. Especialmente ilustrativos son las introducciones de los informes anuales que el Secretario General remite a al Consejo Ejecutivo y a los Estados miembros de la Unesco.

${ }^{2} \mathrm{~A}$ modo de ejercicio de reflexividad. Nuestro propio derrotero de investigación da cuenta de un intento similar. Hacia el año 2009 comenzamos a estudiar la Escuela de Ciencias Políticas de la Flacso y con el tiempo pudimos ponderar el importante aporte de expertos de la Unesco que recibió dicha Facultad. Sin embargo, ¿ese "contingente" de expertos que desembarcaron en la Flacso era significativo en relación a los expertos de la Unesco que circulaban por el resto de América Latina y el mundo? Para avanzar en una respuesta explicativa es que debimos comenzar a pensar a la Unesco en términos relacionales como un espacio de poder, como un campo de disputas interestatales y al mismo tiempo analizar iniciativas de la Unesco en otros países sudamericanos. Esto nos permitió elaborar una explicación del impacto del programa de la Unesco que superara los particularismos de cada una de la iniciativas o intervenciones de la Organización. (ABARZUA CUTRONI, 2015).

${ }^{3}$ La articulación de estos ejes no es un fenómeno privativo de la Unesco. Probablemente podamos observar dichas articulaciones (junto con las tensiones que implican) en otras organizaciones internacionales, especialmente en el sistema de organizaciones que conforman la Organización de Naciones Unidas.
} 
construcción de poder simbólico (BOURDIEU, 1999 [1977], 1999, [1992] 1999, 2013, 2015) tuvieron particular relevancia en la ejecución de su programa.

\section{Cimentar el compromiso inicial}

La relación entre la Unesco y la Flacso comenzó en 1957 y perdura, cambios mediante, prácticamente hasta hoy. En el ideario de la Unesco la Flacso constituía una iniciativa casi perfecta porque permitía que la Organización contribuyera a una iniciativa de carácter regional y por esto desprovista, supuestamente, de particularismos nacionales. Constantemente los funcionarios de la Unesco debían resguardar el carácter universal del programa, porque esta era la fuente de su poder simbólico, y evitar en la práctica críticas por beneficiar más a algunos Estados Miembros que a otros. (ABARZÚA CUTRONI, 2015). Además, la Flacso estaría dedicada fundamentalmente a la enseñanza de las ciencias sociales, lo que coincidía con precisión con la misión asignada a la Unesco según el principio, enunciado una y otra vez por los agentes vinculados a la Unesco, "sembrar la paz en la mente de los hombres”. (Preámbulo, Constitución de la Unesco, 2010).

Todo esto sin el riesgo que implicaba apostar por una institución que no tuviera un fuerte respaldo académico y estatal a nivel local. La experiencia les indicaba a los funcionarios de París que las iniciativas que no gozaban de un fuerte asidero local tendían a diluirse. Cuando en 1957 se elaboró el currículo de ELAS (Escuela Latinoamericana de Sociología de la Flacso) los miembros del departamento de ciencias sociales de la Unesco colaboraron. En relación a esto el Director General señaló en su informe anual que si bien el departamento podía contribuir el éxito de la Facultad "dependía en gran medida del apoyo del gobierno [chileno] y de la cooperación de los medios científicos de los países de la región". (Rapport du Directeur Général, 1957, p. 144)4. Desde un principio tanto el Estado chileno como la Universidad de Chile estuvieron fuertemente comprometidos con la Facultad.

El momento de creación de Flacso es particularmente rico para conocer cómo se articulaban los ejes político-académico y nacional-internacional cuando entraba en juego la posibilidad de un patrocinio de parte de la Unesco. Las negociaciones para la creación de la Facultad comenzaron en 1956. En marzo de ese año se había realizado en Río de Janeiro la "IV Conferencia Regional sobre la Enseñanza de las Ciencias Sociales". Participaron de la misma, representantes de diez países de Sudamérica ${ }^{5}$, organizaciones internacionales, asociaciones científicas/educativas nacionales y un miembro del Departamento de Ciencias Sociales de la Unesco. Entre las resoluciones y recomendaciones de esta conferencia se sugería la creación de una Facultad de Ciencias Sociales en Santiago y un Centro Latinoamericano de Investigación en Ciencias Sociales en Río de Janeiro (CLAPCS) ${ }^{6}$. (Rapport du Directeur Général, 1956, p. 94).

Siete meses después, la Comisión Nacional chilena organizó una mesa redonda para motorizar aquella recomendación en vistas de la próxima realización de Conferencia General de la UNESCO. El staff del Departamento de Ciencias Sociales de la Organización

\footnotetext{
${ }^{4}$ La traducción del francés es nuestra y el añadido también.

${ }^{5}$ Argentina, Bolivia, Brasil, Chile, Colombia, Ecuador, Paraguay, Perú, Uruguay y Venezuela.

6 Recomendamos consultar: GRIZENDI, Ezequiel. El centro de la periferia: internacionalización de las Ciencias Sociales y redes académicas latinoamericanas. Manuel Diegues Junior y los avatares de la sociología del desarrollo. Crítica e sociedade - Revista de Cultura política, v. 4, n. 2, p. 148-167, dez. 2014.
} 
debía cerciorarse de la factibilidad y posterior continuidad de la iniciativa antes de comprometer definitivamente ayuda financiera y expertos. Es decir debía construir los consensos académicos necesarios para el sostenimiento de la futura Facultad. Entonces enviaron en misión a Santiago de Chile, en calidad de experto de la Unesco, a Peter Heintz ${ }^{7}$. Días antes de la mesa redonda, este sociólogo suizo recibe instrucciones de T. H. Marshall (Dir. del Dpto. de Ciencias Sociales) y de K. Szczerba-Likiernik (Jefe de la Div. para el Desarrollo Internacional de las Ciencias Sociales). Heintz debía elaborar un informe detallado sobre la iniciativa y evaluar en qué medida esta contaba con el respaldo suficiente. (Carta de T. H. Marshall a Peter Heitnz, 20 de septiembre de 1956). Desde un principio el patrocinio de la Unesco estuvo supeditado al apoyo conjunto del Estado chileno, la OEA (Organización de Estados Americanos) y la Cepal (Comisión Económica para América Latina). (Carta de Szczerba-Likiernika a Heintz, 28 de septiembre de 1956).

Meses después, los delegados de la $9^{\circ}$ Conferencia General de la Unesco en Nueva Dheli aprobaron un monto de U\$S $120.000^{8}$ destinado a la Flacso en Santiago y el CLAPCS en Río de Janeiro. Este crédito presupuestario inicial no se dividiría en partes iguales sino que la distribución de los montos para cada "centro" "estaría condicionada por las negociaciones del Director General con los dos países interesados". ( $9^{\circ}$ Conferencia General, 9 C/Resolutions: Res. 3.42 d) y Anexo A, punto 3.4, p. 102, 1956). En esa misma Conferencia los destinos de la Flacso y del CLAPCS comenzaron a separarse. La primera institución de ciencia sociales debería dedicarse exclusivamente a la enseñanza mientras que la segunda exclusivamente a la investigación. (Rapport du Directeur Général, 1956, p. 94).

Del 8 al 18 de abril de 1957 se celebró en Río de Janeiro la "Conferencia intergubernamental sobre la enseñanza de las Ciencias Sociales en América del Sur", organizada por el Instituto Brasilero para la Educación, la Ciencia y la Cultura. Durante dicha conferencia, el 17 de abril de 1957, los Estados Latinoamericanos interesados suscribieron los Estatutos del CLAPCS y la Flacso. En relación a la Facultad se decidió que la primera sección que tendría sería una Escuela de Sociología que comenzaría a funcionar en 1958. (Rapport du Director Général, 1957, p. 142).

Casi tres años después del "puntapié inicial" en Río de Janeiro, el 6 de noviembre de 1958, se reunió en la sede de la Unesco en París la "Asamblea Consultiva", conjunta, del CLAPCS y la Flacso. En dicha reunión se acordó que veinte Estados de América Latina harían aportes financieros para el funcionamiento de ambas instituciones de Ciencias Sociales. Para establecer los montos de dichos aportes adoptaron la escala de contribuciones de la Unesco. Tanto Brasil como Chile acordaron contribuir en mayor medida debido a que eran los "países sede". El Director General fue autorizado para recaudar estas contribuciones y distribuirlas entre ambas instituciones. (10 Conferencia General, 10 C/Resolutions: Res. 3.44 y Anexo I, punto 114, p. 113. p. 96, 1958).

La diplomacia chilena trabajó intensamente para sumar adhesiones para Flacso en el seno de la Unesco. Fue especialmente importante el rol de quien sería el primer Secretario General de la Facultad, Gustavo Lagos Matus ${ }^{9}$. (FRANCO, 2007). En

\footnotetext{
7 Sociólogo suizo (1920-1983). PhD de la Universidad de Colonia (1950). En 1956 se desempeñó como experto de la Unesco en Costa Rica y en Colombia.

8 Dólares corrientes.

9 Abogado chileno (1924-2003). Estudió derecho en la Universidad de Chile (1948) y fue docente en esa
} 
consecuencia, a raíz de una propuesta de la delegación chilena, la $10^{\circ}$ Conferencia General autorizó al Director General a: "Participar [...], a petición del gobierno de Chile, en el funcionamiento de la Facultad Latinoamericana de Ciencias Sociales, de Santiago de Chile”. (10 Conferencia General, 10 C/Resolutions: Res. 3.42, 1958).

Beigel (2009) afirma que el "alto patrocinio" de la Universidad de Chile, en el marco de una política sostenida de modernización del sistema científico-universitario chileno, fue decisivo para la creación y sostenimiento de la Flacso a lo largo del tiempo. El 20 de agosto de 1959, se firmó un acuerdo especial para la cooperación de la Unesco-Flacso en el marco del "Acuerdo básico" entre Chile y la Unesco suscrito cuatro años antes. El dinero del Estado chileno era transferido a la Flacso mediante la Universidad de Chile. Esto implicó para los funcionarios de la Unesco una clara señal de que el Estado chileno y la Universidad de Chile podían garantizar la continuidad de la Facultad una vez finalizado en 1969 el patrocinio programado de la Unesco. (13 Conferencia General, $13 \mathrm{C} /$ Resolutions, Res. $3.233,3.234,3.235,1964)$.

\section{Edificar una institución prestigiosa}

Para el período 1959-1970 la contribución financiera de la Unesco a la Flacso fue relativamente importante. Los aportes de la Organización provenientes del Programa de Participación de los Estados miembros sumaron un total de U\$S 574.200. (BEIGEL, 2009, p. 327). Sin embargo, el aporte de la Unesco no se limitó a lo económico. Desde nuestra perspectiva, el valor agregado del patrocinio de la Unesco a la Flacso fue preminentemente simbólico. Durante los primeros años, a partir del otorgamiento de becas y el reclutamiento internacional de expertos, la Unesco contribuyó, junto con la Universidad de Chile, a la construcción del prestigio académico de esta, en aquel entonces, nueva Facultad de ciencias sociales. Con el tiempo, este prestigio académico, multiplicado por la propia "inmersión" de la Flacso en el campo académico chileno, primero, y latinoamericano después, retroalimentaría la legitimidad política de la propia Unesco en América Latina, es decir su poder simbólico en la región.

La Secretaría General de la Unesco tuvo una política decidida de patrocinio a la Flacso chilena ${ }^{10}$. Durante quince años (1958-1973) la Organización reclutó y contrató a unos 21 expertos para que se desempeñaran como docentes-investigadores de la Flacso generalmente durante períodos prolongados de tiempo. (ABARZÚA CUTRONI, 2015, p. 197). Todos estos expertos tenían asignado un mismo objetivo, una misma misión: fortalecer la enseñanza de las Ciencias Sociales en América Latina. La Flacso fundamentalmente tuvo un efecto multiplicador en cuanto a la masa de crítica docentesinvestigadores latinoamericanos dedicados al establecimiento de agendas de investigación ancladas en problemáticas regionales. (FRANCO, 2007; PÉREZ BRIGNOLI, 2008; BEIGEL, 2009; CORTÉS, 2008; STAVENHAGEN, 2014).

misma casa de estudios. En 1969 fue Ministro de Justicia durante el gobierno de Frei Montalva.

10 A nivel regional solo podríamos comparar este nivel de interés en una iniciativa con el Crefal (Centro Regional de Educación Fundamental para América Latina) en Pátzcuaro, México. Esta iniciativa en materia educativa tenía como finalidad mejorar los niveles de alfabetización de la región mediante la formación de educadores (maestros, pedagogos, etc.) para América Latina. Este Centro se creó en 1950 con el patrocinio conjunto de la OEA y la Unesco. Solo en el período 1950-1959 se especializaron en "educación fundamental" un total de 420 personas provenientes de diecinueve países de América Latina, aunque la gran mayoría de los graduados eran mexicanos. 
La Flacso mientras tuvo sede exclusivamente en Santiago de Chile (1958-1973) estuvo conformada por dos escuelas de enseñanza. La Unesco realizó un importante aporte al staff de docente-investigadores de ELAS (Escuela Latinoamericana de Sociología) y secundariamente al de la ELACP (Escuela Latinoamericana de Ciencia Política). El mecanismo de selección y contratación de los expertos destinados a la Flacso en Santiago ${ }^{11}$ da cuenta de cómo se articularon los ejes nacional-internacional y políticoacadémico.

Los futuros docentes podían ser sugeridos por el Estado chileno y/o académicos locales, preferentemente de la Universidad de Chile, o por el Departamento de Ciencias Sociales de la Unesco. Antes de la contratación, los perfiles de los "expertos" eran aprobados por el Estado solicitante. La prerrogativa por excelencia de la Unesco era el reclutamiento del docente y la evaluación de su perfil académico. Este proceso aseguraba que su experticia, en materia de ciencias sociales en el caso de la Flacso, estuviera avalada internacionalmente, es decir, reconocida y certificada, por la Unesco.

\section{Académicos reclutados y avalados por la Unesco}

Durante diez años, la Dirección de la ELAS estuvo a cargo de académicos avalados en su competencia por la Unesco: José Medina Echavarría ${ }^{12}$ (1958-1959), Peter Heintz (1960-1965) y Glaucio A. Dillon Soares ${ }^{13}$ (1966-1968). Luis Ignacio Ramallo Massanet ${ }^{14}$, cuarto director (1969-1973), no nos consta que haya sido contratado como experto de la Unesco para ejercer ese cargo, pero sí fue contratado en 1968 por la Organización como docente de ELAS. (Index of field missions reports, 1947-1968, p. 19, 24). En 1966, cuando se creó la Escuela Latinoamericana de Ciencia Política y Administración Pública (ELACP) su director Horacio H. Godoy ${ }^{15}$ (1966-1973) también fue contratado en calidad de experto de la Unesco. (Rapport du Directeur Général, 1966, p. 82; ABARZÚA CUTRONI, 2012).

El 14 de abril de 1958 comenzaron las clases en la Flacso. Algunos de los primeros cursos fueron dictados por expertos de la Unesco: José Medina Echavarría, Peter Heintz y Lucien Brams. (Rapport du Directeur Général, 1958, p. 92). Los tres eran sociólogos y de origen europeo, aunque al momento de ser enviados a Chile, al menos Medina Echavarría y Heintz, ya conocían otros países de América Latina y la problemática social de la región. Más tarde, en 1959, se sumó al staff otro experto familiarizado con las problemáticas regionales, el antropólogo Alfred Métraux ${ }^{16}$. (Rapport du Directeur Général, 1959, p. 107). Un año después la Organización reclutó para la ELAS otro experto europeo, el sociólogo

\footnotetext{
11 Pudimos asimismo constatar que dicho mecanismo formal se repite en el reclutamiento y contratación de otros expertos enviados a la Argentina y Brasil durante las décadas de 1950 y 1960, aunque con matices diplomáticos establecidos según cuál fuera el Estados miembro que solicitara asistencia técnica.

12 Sociólogo español (1903-1907). Licenciado en derecho y jurisprudencia de la Universidad de Valencia (1924) y doctor en Derecho de la Universidad Central de Madrid (1929). (MORALES MARTíN, 2010).

13 Sociólogo brasilero (1934-). Abogado de la Universidad Cándido Méndez (1957), graduado en sociología y ciencia política de la Pontificia Universidad Católica de Río de Janeiro (1958) y PhD en sociología de la Washington University en St. Louis (1965).

${ }_{14}$ Psicólogo social español. PhD. En Psicología Social de la Universidad de Harvard. Dio clases en Santiago, Asunción, Buenos Aires y París. (FRANCO, 2007).

${ }^{15}$ Funcionario internacional argentino. Abogado de la Universidad de La Plata (1949) y PhD en jurisprudencia de la Universidad de Yale (1961). (ABARZÚA CUTRONI, 2012).

${ }^{16}$ Antropólogo suizo (1902-1963) Se doctoró en La Sorbonne y regresó a la Argentina para realizar estudios de campo. Entre 1928 y 1935 fue el principal impulsor y primer director del Instituto de Etnología de la Universidad Nacional de Tucumán y de la Revista de Etnología. (BEIGEL, 2009).
} 
Stanislav Andreski ${ }^{17}$. (Rapport du Directeur Général, 1960, p. 118). Mientras que en 1963 se sumó al sociólogo noruego Johan Galtung ${ }^{18}$ y en 1965 a la socióloga alemana Renate Maynt $\mathrm{z}^{19}$. Desde 1966, gracias a un acuerdo entre la Unesco y el gobierno sueco, la ELAS contó entre sus profesores a Ingvar Ahman. El mismo año, Werner Ackermann ${ }^{20}$ dictó un curso de psicología social. En 1968, este experto volvió a Chile a cargo de una segunda misión como asesor del director de ELAS. (Index of field missions reports, 1947-1968, p. 23, 24; Index of field missions reports, 1969, p. 5; Rapport du Directeur Général, 1966, p. 82; Ackermann 1966, 1969).

La Unesco contribuyó al proceso de latinoamericanización de la Flacso, proceso que fue impulsado por la cada vez más intensa incorporación de la Facultad Latinoamericana en el politizado campo académico chileno. (BEIGEL, 2009). En 1963, por primera vez la Organización contrató a graduados de la Escuela de Sociología. Uno de ellos fue Edmundo Fuenzalida ${ }^{21}$ (segunda promoción ELAS 1960-1961). Gustavo Lagos, profesor de Fuenzalida en la Facultad de Derecho de la Universidad de Chile, lo había contratado para que colaborara en el primer seminario de Flacso en el año 1958 y lo convenció de estudiar sociología en la ELAS. (FRANCO, 2007). A este experto chileno se le sumaron otros latinoamericanos graduados en la escuela de sociología: en 1963 también, el médico argentino, Juan César García ${ }^{22}$ (segunda promoción ELAS 1960-1961) y luego en 1966, Eduardo Muñoz Ramírez (tercera promoción ELAS 1963-1964). (Index of field missions reports, 1947-1968, p. 24).

Desde 1970 los expertos de la Unesco para la Flacso estarían destinados a la ELACP y al Instituto Coordinador de Investigaciones Sociales (Icis), creado ese mismo año. Atilio Borón ${ }^{23}$ (segunda promoción ELACP) fue contratado como experto para dar clases y realizar tareas de investigación en la escuela de ciencias política. El politólogo argentino ya formaba parte de dicha escuela desde 1969 y continuaría vinculado a esta al menos hasta 1971. También en 1970, Carlos Fortín ${ }^{24}$ fue contratado como experto de la Unesco. Este académico no era diplomado de la ELACP pero había sido miembro "fundador" del staff de la ELACP, Escuela en la que permaneció durante cinco años (1966-1971). En 1971, es contratado para el Icis el sociólogo de la educación Orlando Albornoz Hernández. En 1972, se sumaron al Instituto Bjorn Feuer y a la ELACP Antón de Schutter y Chistian Schoenmakers. (Index of field missions reports, 1970, p. 4-5; 1971, p. 4-5; 1972, p. 6-8;

17 Sociólogo polaco-británico (1919-2007). En 1946, cuando estuvo en Gran Bretaña con el ejército polaco (al que se había reincorporado luego de escaparse de una prisión del ejército soviético) obtuvo un "grado externo" y luego un PhD en la Universidad de Londres.

18 Sociólogo noruego (1930-). En 1956 obtuvo el grado de PhD en matemáticas y en 1957 en sociología. Sobre la "denuncia" de Galtung sobre el "Proyecto Camelot" recomendamos consultar: Navarro y Quesada (2010).

19 Socióloga alemana (1929-). En 1953 obtuvo el grado de doctora en sociología de la Universidad Libre de Berlin. Obtuvo la habilitación docente en la Universidad Libre de Berlin (1957).

${ }^{20}$ Psicólogo alemán (¿1931-2006?). Nacido en Alemania, a los nueve años de edad su familia se instaló en Chile donde cursó todos sus estudios. Graduado en psicología de la Universidad de Chile (1956).

${ }^{21}$ Sociólogo chileno. Abogado de la Universidad de Chile (1959). En 1960 obtuvo el doctorado en filosofía del derecho en la Universidad de Roma.

22 Médico y sociólogo argentino (1932-1984). Referente del movimiento de medicina social en América Latina. Médico de la Universidad de La Plata (1959).

23 Politólogo argentino (1943-). Licenciado en Sociología de la Universidad Católica Argentina (1965) y diplomado de la ELACP-Flacso (1967). En 1976 obtuvo el grado de Doctor en Ciencia Política por la Universidad de Harvard (Cambridge, Massachusetts).

${ }^{24}$ Abogado chileno (1940-). Estudió Derecho en la Universidad de Chile (1962) y es PhD de la Universidad de Yale (1969). 


\section{Estudiantes becados por la Unesco}

Veamos a continuación qué importancia tuvo el otorgamiento de becas Unesco para la conformación de las sucesivas cohortes de estudiantes de la Flacso ${ }^{25}$. Durante el período $1958-1973^{26}$ hubo un total de 293 egresados. A pesar de que la Facultad fue concebida como una iniciativa para mejorar la enseñanza de las ciencias sociales a nivel regional, la distribución geográfica de los diplomados muestra que Chile fue el país más beneficiado, seguido por Argentina, Brasil y México. Estos cuatro países juntos acumularon el $62 \%$ de los graduados, en detrimento de los países del Caribe que tuvieron escasa representación entre los egresados de Flacso. (BEIGEL, 2009, p. 335-336).

Registramos que entre 1958 y 1973 la Unesco becó a 42 personas para que estudiaran en la Flacso (sobre todo sociología), de estas un $76 \%$ (32) obtuvieron sus diplomas. Este grupo representa el $11 \%$ del total de egresados de Flacso. Ahora bien, este porcentaje pequeño oculta la importancia que tuvieron las becas de la Unesco para poner en marcha la ELAS en 1958 cuando aún no gozaba del prestigio que alcanzó luego a nivel regional. El $52 \%$ de las becas Unesco fueron otorgadas a estudiantes de las dos primeras cohortes (1958 y 1960), los cuales en su totalidad finalizaron sus estudios y representan el $54 \%$ del total de egresados de las dos primeras cohortes. (Repertorio de Becarios Unesco 1948-1968, p. 6, 7, 14, 22, 39, 45, 65, 69, 112, 118, 123;1969-1970, p. 3; 1971-1972, p. 18; 1973, p. 51; PÉREZ BRIGNOLI, 2008, p. 280-289).

La distribución geográfica de los becarios Unesco muestra la misma tendencia que los diplomados de Flacso en general. La mayoría de los egresados provenían del cono sur y México ${ }^{27}$. Al momento de ser seleccionados por la Unesco y su Estado de origen ${ }^{28}$ algo más de la mitad de los becarios eran profesores y/o investigadores universitarios, los demás eran funcionarios universitarios o ministeriales y estudiantes que ya eran estudiantes en ELAS. Son llamativos los casos de Anibal Quijano ${ }^{29}$ que era profesor en dos colegios secundarios de Lima y de Diego Palma que era sacerdote. Hubo solo dos becarios Unesco que egresaron de la ELACP. En general las becas eran de larga duración (de 12 a 22 meses), algunas eran de media duración (de 9 a 10 meses). Las del primer tipo cubrían el

${ }^{25}$ Las becas Unesco no fueron las únicas otorgadas para estudiar en FLACSO, fueron muy importantes también las becas de la OEA. (BEIGEL, 2009).

${ }^{26}$ Desde 1974 no registramos becas para la Flacso en ninguna de sus sedes. Existe la posibilidad de que alguna beca haya sido asignada en el marco del PNUD. De todos modos, las becas destinadas a candidatos de América Latina y el Caribe decrecieron considerablemente desde 1974. (ABARZÚA CUTRONI, 2015).

${ }^{27}$ Argentina tuvo 5 becarios de 1958 a 1962, Brasil 4 de 1958 a 1960, Bolivia 1 en 1960, Colombia 3 de 1958 a 1962, Costa rica 1 en 1958, Guatemala 1 en 1960, México 7 - en dos etapas de 1958 a 1962, primero, y de 1970 a 1973 después - , Panamá 1 en 1958, Paraguay 1 en 1962, Perú 4 de 1958 a 1960, Uruguay 3 de 1958 a 1962 y Venezuela 3 de 1962 a 1964. Chile obtuvo también 8 becas Unesco entre 1969 y 1970. Estas fueron financiadas por el PNUD y no por el Programa Participación en el marco del patrocinio programado, debido a que este acuerdo no permitía otorgar becas al país anfitrión. (Repertorio de Becarios Unesco 1948 - 1968, pág. 6, 7, 14, 22, 39, 45, 65, 69, 112, 118, 123; 1969 - 1970, pág. 3; 1971 - 1972, pág. 18; 1973, pág. 51).

${ }^{28}$ Los Estados miembros intervenían en la selección debido a que la financiación provenía mayoritariamente del Programa de Participación de los Estados miembros de la Unesco, financiado con el presupuesto ordinario de la Organización, o el Programa Ampliado Asistencia Técnica de la ONU en el caso de Chile, financiado por la ONU y ejecutado en parte por la Unesco.

29 Sociólogo peruano (1928-). Estudió letras en la Universidad Nacional Mayor de San Marcos, Universidad en la que también obtuvo su doctorado en 1964. 
primer año de estudios en Santiago o la totalidad del ciclo mientras que las del segundo tipo, inferimos, eran solo para comenzar a cursar o finalizar los estúdios. (Repertorio de Becarios Unesco 1948-1968, p. 6, 7, 14, 22, 39, 45, 65, 69, 112, 118, 123; 1969-1970, p. 3; 1971-1972, p. 18; 1973, p. 51; PÉREZ BRIGNOLI, 2008, p. 280-289).

\section{Preservar a la Flacso}

En 1968, la Flacso fue admitida en el Clacso (Consejo Latinoamericano de Ciencias Sociales) - creado en 1967 - solo como "centro miembro honorario" debido a que no contaba con la autonomía institucional necesaria para ser miembro como requería el estatuto de Consejo. En 1969 finalizó la "ayuda programada" de la Unesco a la Flacso. El Director General ya no recaudaría las contribuciones de los países latinoamericanos. Sin embargo, no se trataba solo de que la Flacso careciera de autonomía frente a la Unesco, ya que en 1972 tampoco fue aceptada como miembro pleno de Clacso, sino que carecía de autonomía frente a los gobiernos regionales, es decir no gozaba de autonomía nacional, debido a que era un organismo intergubernamental. (BEIGEL, 2009; Rapport du Directeur Général, 1969, p. 104).

Unos años después, en 1971, la Flacso pasó a ser una: "institución de educación superior regional autónoma, para la enseñanza e investigación en el campo de las ciencias sociales, constituida por los países latinoamericanos y del Caribe en Santiago de Chile, con el patrocinio del Gobierno de Chile" en virtud del acuerdo del 18 de junio de 1971 por la Flacso y la Unesco. Esta fórmula compleja con la que se definió a la Facultad buscaba señalar la autonomía de la Flacso frente a la Unesco al mismo tiempo que se conservaba su carácter regional sin prescindir del patrocinio del Estado Chileno que hasta el momento había sido decisivo. (Rapport du Directeur Général, 1970, p. 117; 1971, p. 150).

Meses después de ratificado este acuerdo ${ }^{30}$, el gobierno de Chile convocó a la primera Asamblea General de la Flacso. Dicha reunión se celebró París, en la Sede de la Unesco. (Rapport du Directeur Général, 1972, p. 164). Como vimos en los apartados anteriores, referido a expertos y becas, a pesar del fin del patrocinio programado, el retiro de la Unesco de la vida académica de la Flacso no fue abrupto sino gradual, ya que Chile siguió solicitando asistencia técnica a la Organización. Diplomáticamente, la Unesco tampoco se retiró por completo.

El golpe de Estado del 11 de septiembre de 1973, perpetrado por el General Augusto Pinochet contra el Presidente Salvador Allende, puso en suspenso las actividades de la Flacso y rompió definitivamente el "laboratorio de ideas" sociales latinoamericanas que se había gestado en Santiago de Chile desde fines de la década de 1950. (GARRETÓN et al., 2005; GARCIA, 2005; BEIGEL, 2010; YOCELEVZKY, 2015). Durante la $93^{\circ}$ Sesión del Consejo Ejecutivo - octubre de 1973 - sus miembros trataron una comunicación de la Comisión Nacional cubana que denunciaba el saqueo de la residencia de Pablo Neruda y "violaciones de los principios de la Declaración Universal de Derechos Humanos cometidos en Chile". A esta denuncia se le sumaban una serie de comunicaciones de organizaciones internacionales, recibidas por el Director General y presidente del Consejo Ejecutivo, que denunciaban también "que en Chile se había producido una grave violación de los derechos

30 Dicho acuerdo que entró en vigor el 19 de junio de 1972 con la ratificación oficial de tres Estados (Chile, Panamá y Cuba). 
de académicos, científicos y educadores, relacionados con instituciones con las cuales había cooperado la Unesco en la ejecución de su programa”. Entonces, a raíz de estas denuncias el Consejo Ejecutivo tomó la decisión 93 EX/8.2. (93 Sesión del Consejo Ejecutivo, 93 EX/8.2, 1973).

Dicha decisión fue producto de un controvertido debate entre aquellos miembros del Consejo Ejecutivo - pertenecientes en su mayoría al bloque soviético - que exigían un "actitud militante de la Unesco" y que en consecuencia se hiciera lugar inmediatamente a la denuncia de la Comisión Nacional Cubana y se difundiera la misma públicamente; y aquellos partidarios - liderados por el representante argentino Atilio Dell'Oro Maini - de una resolución instrumental de la cuestión que remitiera la controversia a la ONU. Durante el debate, el Delegado Permanente de Chile ante la Unesco, Jorge Berguño, invocó el principio de no intervención en los asuntos internos de un Estado miembro y adujo que la defensa de los derechos humanos no está necesariamente entre las competencias de la Unesco. (93 EX/SR 1 - 27, p. 245-273).

Finalmente se consensuó una decisión moderada que aprobó las gestiones que hasta el momento había realizado el Director General, René Maheu, y los instruía para que mantuviera al tanto al Consejo sobre las denuncias recibidas. Esto dio lugar a una serie de informes sobre la violación de los Derechos Humanos en Chile y nuevos debates en la siguiente reunión del Consejo Ejecutivo. (94 EX/SR 1 - 33, p. 315-326; Informe del Director General 94 EX/49; Informe del Comité de Convenciones y Recomendaciones Relativas a la Enseñanza $94 \mathrm{EX} / 50)$.

Un año después de presentada la denuncia por la Comisión Nacional Cubana, en octubre de 1975, la Conferencia General aprobó la resolución 11.31 que requería el inmediato cese de la violación de los Derechos Humanos y libertades fundamentales en Chile e instruía al Director General para que, dentro del límite de sus facultades, actúe para dar cumplimiento a lo decidido por la Conferencia. (18 Conferencia General, Res. 11.31, 18 C/Resolutions, p. 119-120, 1975). En esta, como en otras cuestiones de política internacional, la Unesco no hacía más que adecuarse a lo decidido por la Asamblea General de las Naciones Unidas mediante la resolución 3219 (XXIXX) (noviembre de 1974) "Protección de los Derechos Humanos en Chile".

En relación a la Flacso concretamente, el informe elaborado por el Director General dice que el funcionario encargado de las relaciones con los Estados miembros informó al Director General sobre los ocho detenidos (4 funcionarios y 4 becarios) después del 11 de septiembre:

De los cuatro funcionarios tres han sido puestos ya en libertad y han abandonado el país. El cuarto se encuentra hospedado en una Embajada desde el 14 de octubre de 1973 sin que hasta la fecha se le haya autorizado a salir de Chile. [...] dos de los becarios que se beneficiaron con becas del Banco Interamericano de Desarrollo (BID) han fallecido: el Sr. Jorge Ríos Dalenz ${ }^{31}$, detenido el 11 de septiembre y el Sr. Ignacio Soto Quiroga ${ }^{32}$, detenido el 16 de septiembre, ambos de nacionalidad boliviana. Según información recibida de las autoridades, el Sr. Ríos Dalenz murió

${ }^{31}$ Militante boliviano (?-1973). En 1969 conformó el Partido Demócrata Cristiano Revolucionario (PDCR), este nuevo agrupamiento junto con otras formaciones políticas sentó las bases del MIR (Movimiento de Izquierda Revolucionaria) boliviano. En agosto de 1971 se exilió en Chile a causa del Golpe Militar de Banzer. Allí se dedicó a estudiar en la ELACP-Flacso y consolidar las relaciones internacionales del MIR boliviano. (FRANCO, 2007).

32 Militante boliviano (?-1973). Abogado, estudiante de la ELACP-Flacso y miembro del MIR boliviano. 
en un intento de fuga y el Sr. Soto Quiroga falleció como consecuencia de heridas que se causó al lanzarse de una ventana para eludir ser interrogado. Los otros dos becarios detenidos fueron liberados y autorizados a abandonar el país en octubre de 1973 y enero de 1974 respectivamente. (94 EX/49, p. 5).

Esta versión oficial ocultaba que en realidad Ríos Dalenz fue detenido en su domicilio y acribillado en la vía pública, luego de ser torturado, y que Soto Quiroga fue detenido y trasladado al Comando de Ingenieros de Chile, donde fue interrogado bajo tortura y después arrojado al vacío desde la azotea del edificio. (FRANCO, 2007).

Según Pérez Brignoli (2008), durante la Asamblea General Extraordinaria de la Flacso (Quito, 1975) la mediación de funcionarios de la Unesco ante la dictadura chilena, fue uno de los factores que permitió la reforma estatutaria necesaria para que la Flacso profundizara el proceso de descentralización iniciado con la creación de la sede en Buenos Aires en 1974. A la vez que se mantuvo un grupo de trabajo en Santiago a partir del "paraguas" institucional que brindaba la Flacso en su calidad de organización académica regional. Un nuevo informe ${ }^{33}$ del Director General de la Unesco al Consejo Ejecutivo da cuenta de que este grupo de trabajo se desempeñaría en el Icis. (97 EX/33, p. 23). Poco después, en 1975 se crearon las sedes de Flacso en México y Ecuador.

En 1977, la Flacso obtuvo un importante reconocimiento de su calidad académica de parte de la Naciones Unidas a través de la Cepal (Comisión Económica para América Latina) y la Unesco. Ese año la Facultad obtuvo el estatus de organismo consultor ante la Cepal y firmó un nuevo acuerdo con la Unesco no ya de patrocinio si no de cooperación. En adelante la Flacso seria consultada por la Unesco en relación a problemáticas vinculadas a la Ciencias Sociales en América Latina y ambas instituciones se comprometían a coordinar sus actividades en la región. Dicho acuerdo no excluía la posibilidad de asistencia técnica, pero a diferencia de los años anteriores este tipo de cooperación entre la Flacso y la Unesco sería recíproca, en vez de unilateral. (Informe de misión, 1986, p. 2; Acuerdo Flacso-Unesco, 1977).

En este marco la Unesco ejecutó una importante ayuda financiera, proveniente de un proyecto regional del PNUD (Programa de las Naciones Unidas para el Desarrollo), que contribuyó a consolidar la descentralización de la Flacso. Registramos que desde 1978 hasta 1983 el Proyecto RLA/78/001 trasfirió un total de U\$S 1.146.217 34 a las sedes de la Facultad para el desarrollo de actividades de docencia e investigación y el otorgamiento de becas. (Informe de misión PNUD-Unesco, 1986, p. 5, 20). Complementariamente, entre 1978 y 1980 el Gobierno ecuatoriano, la Unesco y la Flacso-Quito coordinaron actividades para la puesta en marcha de otro proyecto PNUD (ECU/78/014) que tenía como finalidad el fortalecimiento de las Ciencias Sociales en ese país y aportó la suma de U\$S $619.874^{35}$. (Informe de misión, 1981, p. 3).

En última instancia, el proceso de descentralización y autonomía consolidó el prestigio académico de la Flacso a nivel regional, lo que permitió, entre otros factores, la supervivencia de esta ya institucionalizada Facultad de Ciencias Sociales en América Latina. En 1984 la Unesco debió enfrentar su propia crisis político-institucional. El retiro de

\footnotetext{
${ }^{33}$ Denominado "Situación de la ciencia, la educación, la cultura y la información en Chile: informaciones recibidas e informe de la misión enviada por el director General a Chile (97 EX/33, 25 de abril de 1975).

34 Dólares corrientes.

35 Dólares corrientes.
} 
Estados Unidos y el Reino Unido, en su calidad de miembros de la Unesco, cuestionó fuertemente el poder simbólico de la Organización a nivel internacional y puso en jaque su capacidad económica de otorgar fondos para la ejecución de proyectos fuera de Sede ${ }^{36}$. Sin embargo, dichas críticas tuvieron poco impacto en el prestigio de la Unesco en América Latina. En la actualidad la cooperación entre académicos latinoamericanos y la Organización sigue vigente, sobre todo a través de las actividades del lesalc (Instituto internacional para la Educación Superior en América Latina y el Caribe) y las Cátedras Unesco.

\section{Conclusiones}

A lo largo del artículo intentamos mostrar cómo fue mutando la relación FlacsoUnesco, del patrocinio a la cooperación. Desde 1957 y durante todo el tiempo que se desarrolló esta alianza estratégica, la Unesco, esa inmensa organización intergubernamental de la Naciones Unidas, multiplicó su poder simbólico en América Latina. Esto, en parte debido a haber patrocinado, es decir haber contribuido económicamente, y sobre todo avalado técnicamente y respaldado diplomáticamente a la Flacso y, más adelante, a partir de reconocer un vínculo de cooperación entre instituciones pares. Con el tiempo la Facultad se había consolidado como una institución de enseñanza e investigación de nivel superior que había alcanzado, sobre todo desde su integración al campo académico, chileno primero y latinoamericano después, un importante reconocimiento académico. Todo indica que, la formación de referentes académicos de la Ciencias Sociales en el ámbito de la Flacso y las agendas de investigación que estos desarrollaron a lo largo de sus trayectorias profesionales, tuvieron un impacto positivo en la consolidación de la Ciencias Sociales de nuestra región.

Probablemente el prestigio y la legitimidad del que goza la Unesco en América Latina en la actualidad están cimentados en gran medida en su histórico vínculo con la Flacso. En el 2008, estas dos instituciones firmaron un convenio complementario al acuerdo de 1977. (186 EX/Decisions, 38, 2011). Lo que nos permite inferir que hoy la relación Flacso-Unesco sigue plenamente vigente. En consecuencia, una vez establecida (al menos en parte) la génesis del poder simbólico de la Unesco en América Latina, nos preguntamos: hoy ¿cuáles son las fuentes de la reproducción de dicho poder, de "hacer ver y de hacer creer, de confirmar o trasformar la visión del mundo" (BOURDIEU, 1999 [1977]), de la Unesco en un mundo globalizado?

\footnotetext{
36 Los motivos del retiro de los Estados Unidos y el Reino Unido de la Unesco merecen un análisis pormenorizado en otro artículo. Pero brevemente podemos referir que se trató de un incidente diplomático que pone en evidencia los límites de una Organización como la Unesco para enfrentarse a los intereses de sus Estados miembros más poderosos. En 1978 la Unesco lanzó una declaración sobre los medios masivos de comunicación que abogaba por una distribución más democrática de los canales de distribución de la información. Luego en 1980 la Unesco publicó el mentado "Informe Mc Bride". En el mismo la comisión internacional de expertos que lo elaboró dejaba en evidencia las asimetrías que había en la propiedad de los medios de comunicación a escala mundial. Con esta denuncia Estados Unidos vio amenazados sus intereses comerciales y comenzó una campaña de desprestigio contra la Unesco, a la que acusó de patrocinar el control estatal sobre los medios de comunicación en detrimento de la libertad de expresión. Esto a pesar de que el citado informe no proponía ningún tipo de censura sobre la actividad periodística. Para comprender mejor la relación Estados Unidos - Unesco y sobre el incidente provocado por el informe Mc Bride recomendamos consultar: PRESTON JR, William; HERMAN, Edward S.; SCHILLER, Herbert I. Hope and folly. The United States and Unesco (1945-1985). Minneapolis: University of Minessota Press, 1989.
} 


\section{Fuentes documentales}

ACKERMANN, W. $(1966,1969)$ Informes de misión. Unesco Archive, París.

Actas de la Conferencia General: 9 C/Resolutions, 10 C/Resolutions, 13 C/Resolutions, 18 $\mathrm{C} /$ Resolutions. Unesco Archive, París.

Actas del Consejo Ejecutivo: 93 EX/Decisions, 93 EX/SR 1 - 27, 94 EX/SR 1 - 33, 186 EX/Decisions. Unesco Archive, París.

Acuerdo Flacso-Unesco (1977). 102 EX/26. Unesco Archive, París.

Asamblea General de las Naciones Unidas. Resolución 3219 (XXIXX). Archivo ONU.

Carta de K. Szczerba-Likiernika a Peter Heintz, París, 28 de septiembre de 1956. Carpeta: 370.23 (83) 37.4 "56" 01 AMS. Unesco Archive, París.

Carta de T. H. Marshall a Peter Heitnz, París. 20 de septiembre de 1956. Carpeta: 370.23 (83) 37.4 "56" 01 AMS. Unesco Archive, París.

Constitución de la Unesco (2010). Manual de la Conferencia General. Unesco: París. Index of field missions reports: 1947 - 1968, 1969, 1970, 1971, 1972, 1973. Unesco Archive, París.

Informe de misión (1981). Apoyo a las actividades en Ciencias Sociales para el Desarrollo. Unesco Archive, París.

Informe de misión PNUD-Unesco (1986). Facultad Latinoamericana de Ciencias Sociales. Unesco Archive, París.

Informes de la Secretaría General: 94 EX/49, 94 EX/50, 97 EX/33. Unesco Archive, París.

Rapport du Directeur Général sur l'activité de l'organisation en: 1956, 1957, 1958, 1959, 1960, 1966, 1969, 1970, 1972. Unesco Archive, París.

Repertorio de Becarios Unesco: 1948-1968, 1969-1970, 1971-1972, 1973. Unesco Archive, París.

\section{Referencias}

ABARZÚA CUTRONI, Anabella. Funcionarios internacionales, sus capitales, disposiciones y experticias: la trayectoria de Horacio H. Godoy (1944-1978). Sociedad hoy, Departamento de Sociología, Universidad de Concepción, Chile, n. 22, p. 61-78, $1^{\circ}$ Sem. 2012.

. Un sitio para los imperialismos de lo universal. La Unesco como espacio de disputas inter-estatales (1945-1984). Mayo de 2015. Tesis doctoral - Facultad de Ciencias Sociales de la Universidad de Buenos Aires, 2015.

BEIGEL, Fernanda. La Flacso chilena y la regionalización de las ciencias sociales en América Latina (1957-1973). Revista Mexicana de Sociología, México, D. F., v. 71, n. 2, p. 319-349, 2009.

. La teoría de la dependencia en su laboratorio. In: BEIGEL, Fernanda (Dir.).

Autonomía y Dependencia Académica. Universidad e investigación científica en un circuito periférico: Chile y Argentina (1950-1980). Buenos Aires: Biblos, 2010. p. 129-144. BOURDIEU, Pierre. Do imperialismo de lo universal. In: BOURDIEU, Pierre. Intelectuales, Política y Poder. Buenos Aires: Eudeba, [1992] 1999.

La Nobleza de Estado. Buenos Aires: Siglo XXI, 2013.

Meditaciones pascalianas. Barcelona: Anagrama, 1999. 
. Sobre el Estado. Barcelona: Anagrama, 2015.

. Sobre el poder simbólico. In: BOURDIEU, Pierre. Intelectuales, Política y Poder. Buenos Aires: Eudeba, [1977] 1999.

CORTÉS, Fernando. Los métodos cuantitativos en las ciencias sociales de América Latina. Iconos - Revista de Ciencias Sociales, Facultad Latinoamericana de Ciencias Sociales, Quito, Ecuador, n. 30, p. 91-108, enero 2008.

FRANCO, Rolando. La Flacso clásica (1957-1973). Santiago de Chile: Catalonia, 2007.

GARCIA, Afrânio. Circulation internationale et formation d'une "école de pensée" latinoaméricaine (1945-2000). Social Science Information/Information sur les sciences sociales, v. 44, n. 2/3, p. 521-555, 2005.

GARRETON, Manuel Antonio; MURMIS, Miguel; DE SIERRA, Geronimo; TRINDADE, Helgio. Social sciences in Latin America: a comparative perspective Argentina, Brazil, Chile, Mexico and Uruguay. Social Science Information, v. 44, n. 2 \& 3, p. 557-593, 2005.

GRISENDI, Ezequiel. El centro de la periferia: internacionalización de las Ciencias Sociales y redes académicas latinoamericanas. Manuel Diegues Junior y los avatares de la sociología del desarrollo. Crítica e sociedade - Revista de Cultura política, v. 4, n. 2, 2014.

MORALES MARTíN, Juan Jesús. José Medina Echavarría. Un clásico de la sociología mexicana. Desacatos, n. 33, p. 133-150, mayo/ago. 2010.

NAVARRO, Juan José; QUESADA, Fernando. El proyecto Camelot (1964-1965). La dependencia académica entre el escándalo y el mito. In: BEIGEL, Fernanda (Dir.).

Autonomía y Dependencia Académica: Universidad e investigación científica en un circuito periférico - Chile y Argentina (1950-1980). Buenos Aires: Biblos, 2010, p. 145168.

PÉREZ BRIGNOLI, Héctor. Los 50 años de la Flacso y el desarrollo de la Ciencias Sociales en América Latina. San José, Costa Rica: Editorial Juricentro, 2008.

STAVENHAGEN, Rodolfo. Flacso, Clacso y la búsqueda de una sociología latinoamericana. Perfiles Latinoamericanos, Facultad Latinoamericana de Ciencias Sociales, México D.F., n. 43, p. 7-17, ene./jun. 2014.

YOCELEVZKY, Ricardo. Una nota sobre el desarrollo de las ciencias sociales en América Latina. Perfiles Latinoamericanos, Facultad Latinoamericana de Ciencias Sociales, México D. F., n. 45, p. 203-222., ene./jun. 2015.

ANABELLA ABARZÚA CUTRONI es Doctora en Ciencias Sociales de la Universidad de Buenos Aires. Actualmente es becaria postdoctoral del Instituto de Ciencias Humanas, Sociales y Ambientales (Incihusa) - Consejo Nacional de Investigaciones Científicas y Técnicas (Conicet) en Argentina, profesora JTP de Metodología para la Investigación en Ciencia Política en la UNCuyo y profesora titular de Sociología II en la Universidad de Congreso. Su más reciente publicación es: Partículas universales: las misiones científicas de la Unesco en Argentina (1954-1966) en Revista Iberoamericana de Ciencia, Tecnología y Sociedad - CTS, n. 36., v. 12, octubre de 2017.

Dirección postal: Dr. Moreno, 1228 - Dpto. 2, Las Heras, 5539, Mendoza, Argentina.

E-mail: a.abarzuacutroni1983@gmail.com 
Recebido em 22 de março de 2017.

Aceito em 04 de maio de 2018.

(c) (i) 